\title{
Research on Key Technologies of Multi- Computer Cooperative Virtual Training System Development
}

\author{
Xuping Wang, Chengwei Jiang* and Xingwu Kang \\ Xi'an High-tech Research Institute, Shanxi 710025,China \\ *Corresponding author e-mail: donggim8774116@163.com
}

\begin{abstract}
The key technology of multi-machine (computer) cooperative operation under the specific virtual operating environment of LAN is studied for the maintenance operation of large complex mechanical equipment, which requires multiple people to cooperate and at the same time and place. The design and hardware configuration of the system are determined by the actual requirements of the maintenance operation in the background of the equipment, and finally the LAN environment is built under the support of Unity3D/network technology. On the network module built-in packaged component algorithm, by adding counters and controls the script call implements the optimization of the algorithm, thereby reducing the network delay from the server to the client in the multi-machine collaboration process. The result proves that it is completely feasible to develop a multi-machine cooperative virtual training system of a mechanical equipment based on the optimization of network encapsulation algorithm. The functional design of the system fully meets the actual needs.
\end{abstract}

KEYWORDS: Network, count, multi-computer collaboration, virtual simulation

\section{Introduction}

Due to the high structural complexity of large-scale complex mechanical equipment, when the mechanical equipment is in a state failure, the maintenance support personnel must be able to carry out mechanical equipment on the basis of the inherent knowledge of the entire mechanical equipment and the familiarity of the operating procedures. Troubleshoot and repair, and the entire maintenance support process requires several operators to work together. Therefore, it is necessary to carry out the development and research of the mechanical equipment multi-machine collaborative virtual training system, which not only can control the training cost of the entire maintenance personnel, but also greatly improve the overall maintenance training efficiency of the equipment. As a development platform for virtual simulation environment, Unity3D is powerful and easy to operate. It is widely used 
in the development of virtual simulation systems in large-scale mechanical equipment $[1,2]$. Its built-in network module is basically used in LAN environment. Multi-computer collaborative system development [3, 5]. Unity3D / network technology has a very deep application foundation and practical experience in the field of mechanical equipment, so the key technology research on hardware configuration and collaborative algorithm implementation of multi-machine collaborative system based on Unity3D platform has great Value.

\section{The study of system design}

The multi-computer collaborative virtual training system based on a certain type of mechanical equipment is designed to address the shortcomings of the current large-scale mechanical equipment maintenance support training mode in the industrial field. By providing a virtual training and training platform, the maintenance support personnel can be in the simulated virtual environment. The principle knowledge of mechanical equipment has a deep understanding, and equipment maintenance operation training can be carried out independently or in cooperation with each other, so as to improve the training level and training efficiency, and at the same time reduce the wear and tear on the missile equipment. Therefore, the design mode of the system is mainly the "training + assessment" mode. The training and assessment modules are independent and related to each other. The participants in the system training module are familiar with the internal principles of mechanical equipment operation, and at the same time reach a certain level. At the operational level, the ability to operate and the ability of the maintenance support personnel to cooperate can be assessed. In the training process, auxiliary content such as text, audio and video can be added to improve the simulation authenticity and human-computer interaction of the system. Through continuous design and development of training content, a complete assessment subject system is gradually formed. The assessment mainly records the time of virtual operation and the correct number of errors in operation, and stimulates the training enthusiasm of the participants through an intuitive time concept. The design of the system is shown in Figure 1. 


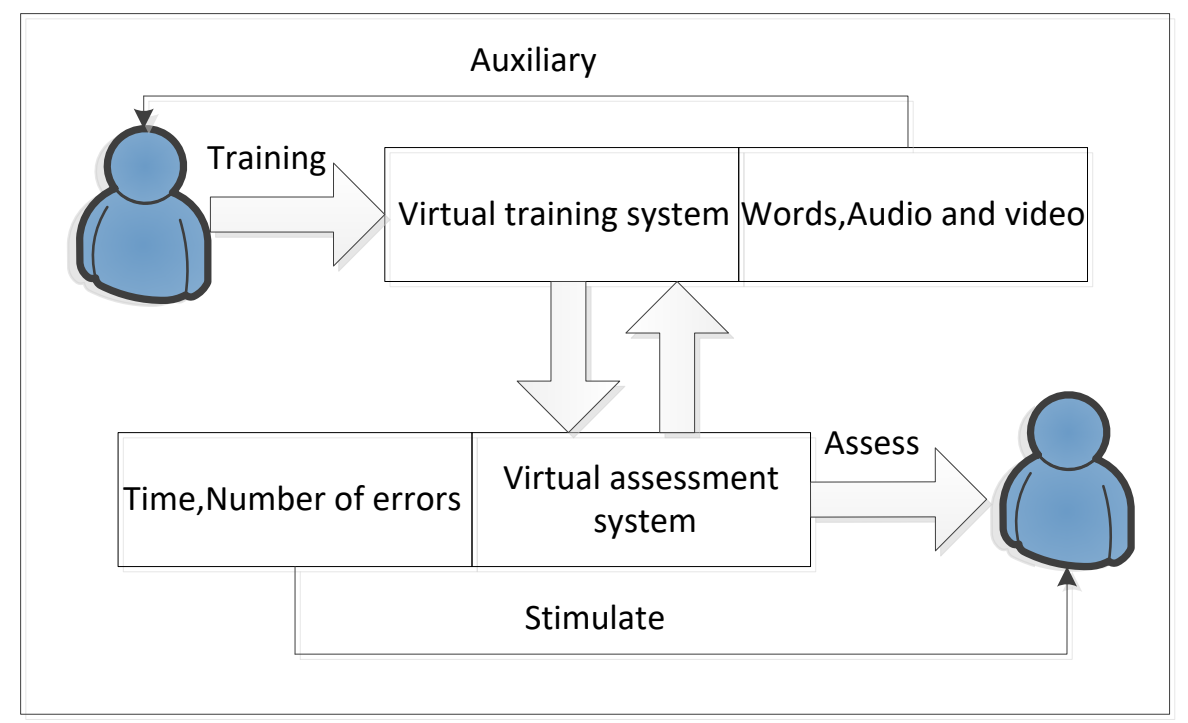

Figure. 1 System design

\section{Hardware Requirements}

\subsection{Network Topology}

The network topology of the multi-machine collaborative system adopts a star topology, and the network center server end is the command center of the virtual maintenance operation of the entire mechanical equipment, and the overall process of cooperative operation is displayed through the projection screen. The other operations are clients. The client can be configured with only one computer. It can also be configured with several computer hosts to establish rendering nodes and collision detection nodes. The specific hardware configuration depends on the simulation object and data transmission volume of the system. At the same time, in order to improve the overall performance of the system, one switch can be configured for each operation position, instead of one switch for the entire system. The network topology of the multi-machine collaborative system is shown in Figure 2.

The system uses a star topology with the following main benefits:

(1) The operation post is easy to expand and easy to move. In the star topology, if the operation position is increased or decreased, it is only necessary to increase or remove the connection of the corresponding network cable to the switch. Unlike a ring network topology, the expansion and movement of devices affect the operation of other node devices in the network. 
(2) The system is easy to maintain in the later stage and the maintenance cost is low. Because of the star topology, each operation post is independent of each other. When a node's computer or network switch fails, other operations can continue to perform virtual training of the stand-alone system. Separate removal and replacement of the failed node does not result in a paralysis of the overall coordination system.

(3) Reduce data traffic and reduce system bandwidth. In the network topology of the system, all the clients and servers have a network switch, which avoids the broadcast mode of information transmission in the LAN environment when the system shares a network switch, and the server-side instructions are sent to each client. In turn, the transmission of redundant data in the system is increased, and the bandwidth of the system is increased.

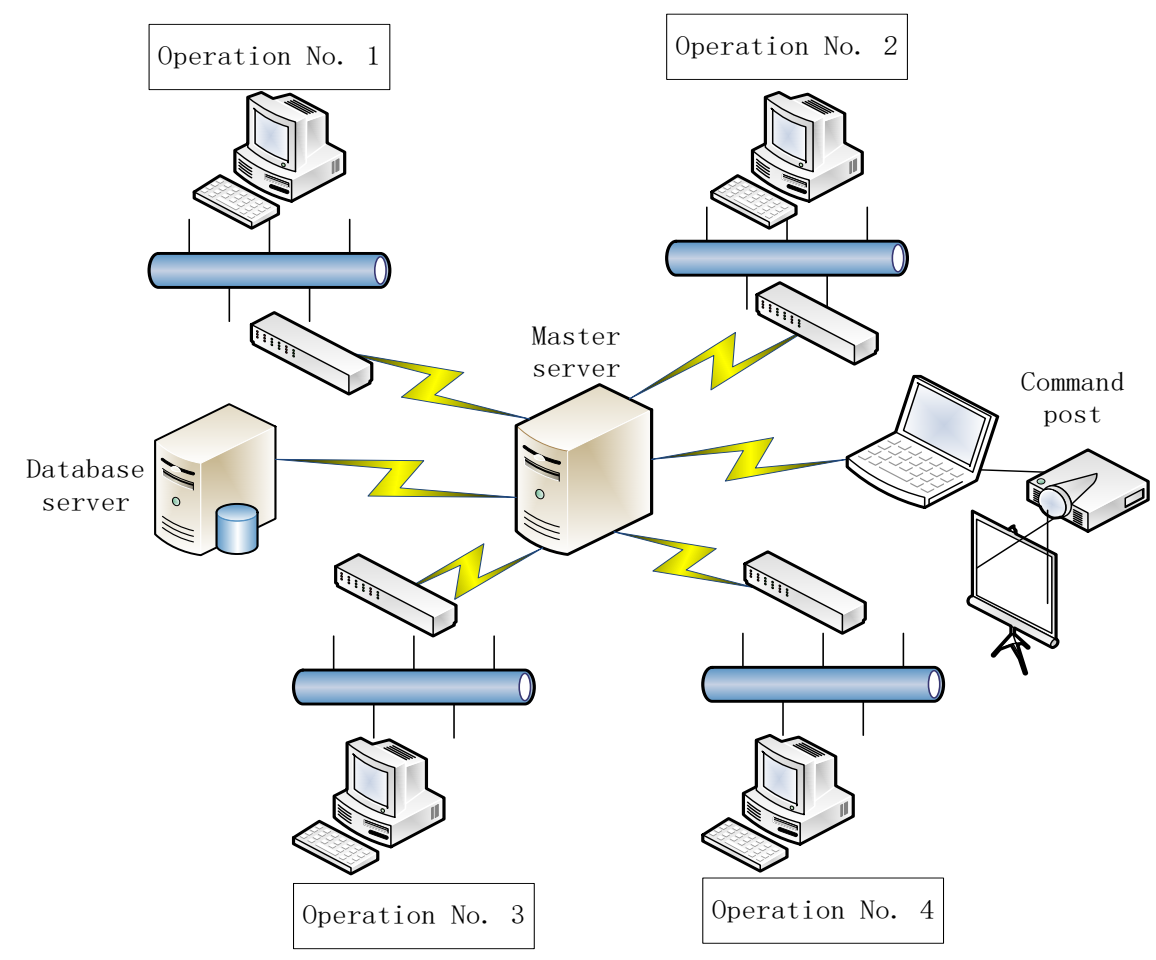

Figure. 2 Network topology

\subsection{The Minimum Configuration of Hardware}

According to the system network topology diagram of Figure 2, combined with the actual situation of mechanical equipment operation and maintenance, all node computers increase the difficulty of hardware configuration and increase the development cost of the system. Therefore, the algorithm in Unity3D built-in 
network module is optimized and implemented. It is especially important to improve the overall performance of the system. On the basis of the optimization algorithm, there is no need to configure too many node computers, and only need to be equipped with ordinary client terminal computers. Therefore, the minimum hardware configuration of the system is a server computer and a client computer and a common peripheral device, and the projection display device is configured on the server computer to facilitate the overall control of the collaborative operation of the command post. Table 1 shows the hardware configuration table for the command post computer and the general post computer.

Table 1 Computer hardware configuration

\begin{tabular}{|c|c|c|}
\hline Job category & Sever (command post) & Client (ordinary post) \\
\hline System & Window7 & Window7 \\
\hline RAM & 8GB & 4GB \\
\hline hard disk & 500GB 7200r & 500GB 7200r \\
\hline Graphics card & 2GB & 1GB \\
\hline
\end{tabular}

\section{Optimization and Implementation of Collaborative Algorithm}

\subsection{The idea of the algorithm}

Under the condition that the actual operation of large-scale mechanical equipment is empty at the same time, the multi-machine cooperative virtual operation under the internal LAN environment is studied. The most important feature of the LAN environment is that the model of the mechanical equipment can be placed on each client computer. Avoid data transfer from the server to the client after the model attribute is updated, and most of the unnecessary data is also involved, thereby increasing the network delay of the client during system operation. The main idea and improvement of the algorithm is to give each operator a specific operation action based on the built-in model data of each client, and judge the step of the operation step through the counter, and pass the operation content. The command is sent to the corresponding client, the client's operation is synchronized to the server, and then synchronized to other clients. Updating only the underlying model of the operation in the local area network is equivalent to the same action triggered by one instruction of several computers that need to be operated, while other model group data does not need to be updated.

In this paper, we add the count on the basis of the synchronization function of the built-in transform component encapsulation algorithm of the network module. The following is a description of the state of the count in the two-dimensional coordinates for the multi-machine coordination step, as shown in Figure 3 and the specific operation mode: 
ISSN 2616-5775 Vol. 1, Issue 1: 71-79, DOI: 10.25236/AJCIS.010007

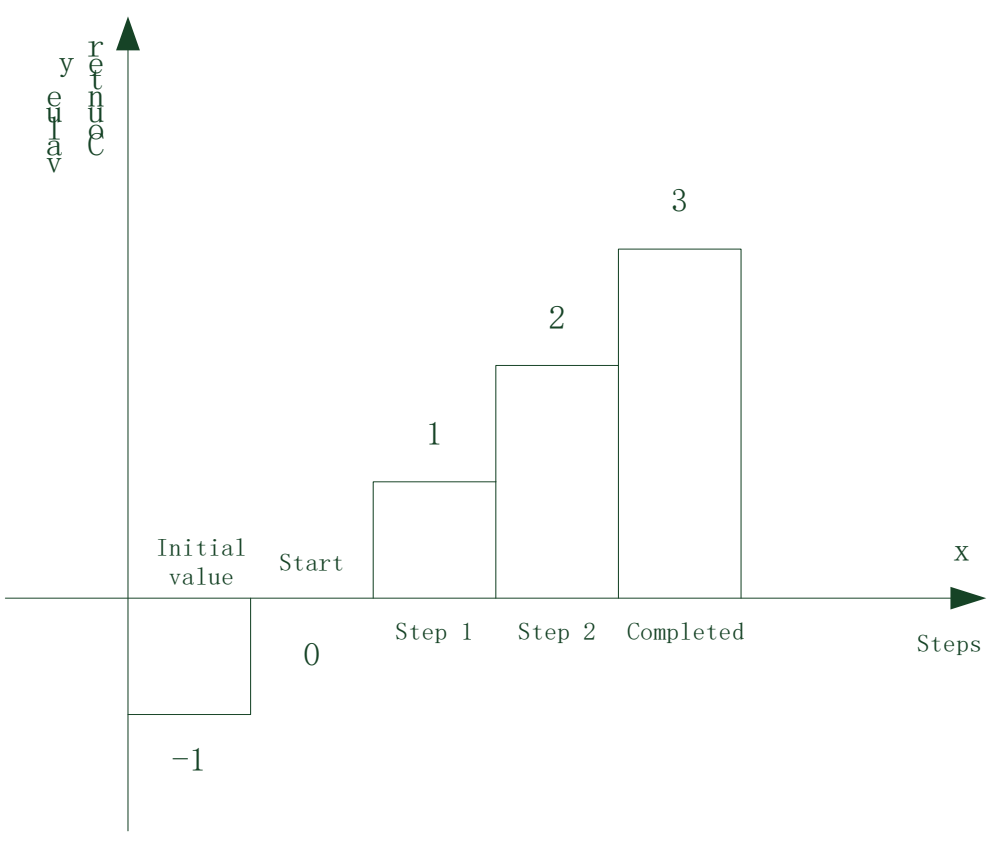

Figure. 3 Counter state diagram

The initial value of count is set to -1 . When all three users $\log$ in to the client, count plus 1 becomes 0 . The system prompts: the first user is ready to operate. The logo multi-machine cooperative operation begins. By analogy, a step is operated, and the count value is incremented by 1 . After all operations are completed, count is incremented by 1 , and the corresponding instruction is called. The system prompts: the coordinated operation is completed.

\subsection{Implementation of the algorithm}

(1) count implementation and control script call

The function and operation mode of count have been analyzed in detail. The actual addition of count is not difficult, but the effect of count alone can not be used to send commands and control the operation of the trumpeter. Count must be related to the control script control and network module. The component determines the logical relationship in order to optimize the algorithm.

First of all, the purpose of count is analyzed. The main function of count is to send an instruction to inform all the hands of the trumpeter to proceed to which step, which trumpeter is required to operate. So the value of count corresponds to a different instruction, the instruction is as follows: 


\author{
if (count $==1)$ \\ \{ShowMsg("User 1 completes the first step "); \\ ShowMsg("User 2 prepares for the second step "); \\ if (count $==2$ ) \\ \{ShowMsg("User 2 completes the second step "); \\ ShowMsg("User 3 prepares for the third step "); \\ if (count == 3) \\ \{ShowMsg("User 3 completes the third step "); \\ ShowMsg("Completing the collaborative operation ");
}

At the same time count must be combined with the network module component to determine the order of operations and the personnel of the operation, and synchronize the counting information to the server, counting one step after the operation. The corresponding code is implemented as: [SyncVar(hook = "AddCount")]

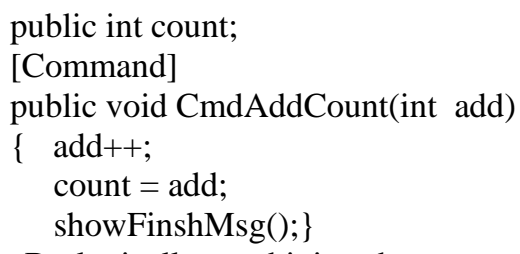

By logically combining the upper and lower two pieces of code, the function of sending a command using count is provided. Count is the basis of the entire algorithm, the specific use of count is called by the control script.

The role of Control is equivalent to the role of an intermediate medium, which calls count, which is also closely connected to the network component, and also controls the behavior of the operation trumpeter.

(2) The actual combination of control script and count

A single implementation of Count and control can be debugged via VS, but specific functional applications need to be done on the network module. At this point, the network plays the role of the main platform. In the first section, the functions of the network module components have been studied in detail. After the components of the network module are added, first add count in the equipment model information bar, and count the initial value is set to -1 . The control implements the specific operation by calling count, so the control script is fixed to each user, so that the command can be sent to each user when the value of count changes. At this time, the data synchronized internally by the server is only the value of count and the corresponding instruction, and there is no transmission of the model data.

The actual combination takes a specific mechanical equipment as an example. The cooperative operation of the tail model requires three operators to cooperate. The element 0 indicates that the corresponding operator player_1 needs to perform the operation on the base model A_001 when the count is 0 . The remaining steps are performed. And so on. Figure 4 shows the parameter settings during the actual combination of count and control.

\title{
Published by Francis Academic Press, UK
}




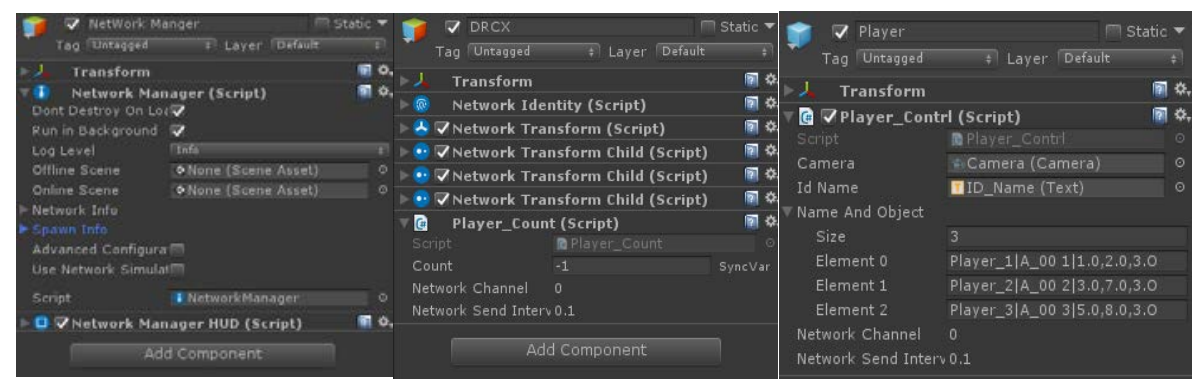

Figure. 4 Parameter setting of multi computer cooperative algorith

\section{Simulation Verification}

Compared with the network-encapsulated algorithm, the optimization algorithm proposed in this paper adds count, and changes the way the model and operation steps are synchronized, which reduces the transmission of redundant model data. On the framework of the system design scheme, the hardware configuration is built, and on this basis, the multi-machine collaborative virtual training prototype system of the mechanical equipment is developed through the Unity3D platform. In the case that the optimization algorithm greatly reduces redundant data transmission, the performance of the entire prototype system is good and the model rendering is smooth. Figure 5 shows the operation behavior of the command post operator and the general operator in the virtual environment under a certain operation step.

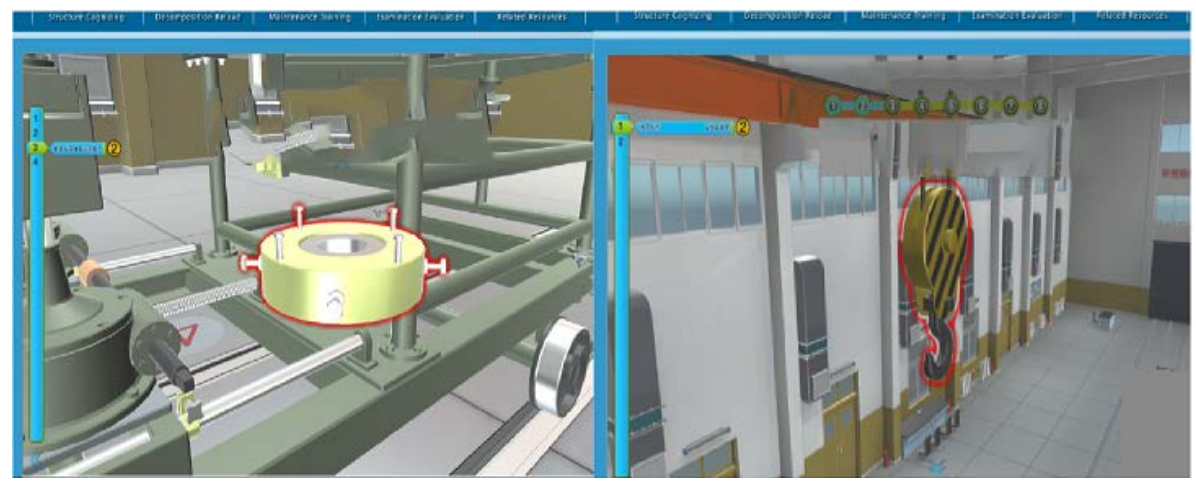

Figure. 5 Operational behavior demonstration

\section{Conclusion}

Based on the detailed research on the key technologies of multi-machine collaborative development in LAN environment, this paper firstly designs and designs the system design and hardware minimum configuration. Finally, the

\section{Published by Francis Academic Press, UK}


ISSN 2616-5775 Vol. 1, Issue 1: 71-79, DOI: 10.25236/AJCIS.010007

original encapsulation algorithm is optimized, through the addition of count and the control script. The algorithm is optimized and the purpose of the algorithm is improved, so that the network delay from the server to the client in the multimachine cooperation in the LAN environment is much less. The simulation experiment is carried out to verify the data transmission efficiency of the optimization algorithm and the feasibility of the key technology of the system development. The results show that the optimization algorithm under the system design scheme has certain feasibility and can be applied to the virtual multi-machine of large mechanical equipment collaborative operation.

\section{References}

[1] LUAN F. Development Of Virtual Simulation Teaching System For Hydraulic Transmission Based On Unity3D [D]. Ji'nan: Shandong University of Architecture and Technology, 2015.

[2] YANG Y B, LI M, XIE H W. Solutions Of Desktop Virtual Maintenance Training System Based On Unity3D [J]. Journal of The Aapplication of Computer, 2016, 36 (S2): 3.

[3] CHEN J F. Research And Implementation Of Unity3D Based Cross Platform Mobile Phone Network Games [D]. Guangzhou: Zhongshan University, 2013.

[4] FENG Y Q. Research On Collaborative Work Platform For Maintenance And Disassembly Process [D]. Wuhan: Huazhong University of Science and Technology, 2014.

[5] HUANG P. Research And Implementation Of Distributed Collaborative Virtual Assembly Simulation Based on Unity3D [D]. Xiangtan: Xiangtan University, 2017. 